\title{
Feiras livres e a capilaridade das cadeias curtas em Porto Grande - AP
}

\author{
Open fairs and the capilarity of short chains in Porto Grande - AP \\ Ferias abiertas y capilaridad de las cadenas cortas en Porto Grande - AP
}

Recebido: 02/09/2021 | Revisado: 09/09/2021 | Aceito: 14/09/2021 | Publicado: 16/09/2021

\author{
Raquel Nominato Araújo \\ ORCID: https://orcid.org/0000-0002-5075-3268 \\ Instituto Federal de Educação, Ciência e Tecnologia de Sergipe, Brasil \\ E-mail: raquel.araujo@ifs.edu.br \\ Najla Kauara Alves do Vale \\ ORCID: https://orcid.org/0000-0002-3577-1513 \\ Instituto Federal de Educação, Ciência e Tecnologia Goiano, Brasil \\ E-mail:najla.vale@ifgoiana.edu.br \\ Cleide Ane Barbosa da Cruz \\ ORCID: https://orcid.org/0000-0002-8277-1460 \\ Centro Universitário Estácio de Sergipe, Brasil \\ E-mail: cleianebar@gmail.com \\ Wanusa Campos Centurión \\ ORCID: https://orcid.org/0000-0002-2822-7392 \\ Instituto Federal de Educação, Ciência e Tecnologia de Sergipe, Brasil \\ E-mail: wanusa.centurion@ifs.edu.br
}

\begin{abstract}
Resumo
A capilaridade das cadeias curtas agroalimentares possibilita a geração de benefícios econômicos tanto para produtores quanto consumidores. Por isso, esta pesquisa tem como objetivo averiguar a capilaridade das cadeias curtas agroalimentares, instituída na feira de agricultura familiar no município de Porto Grande - AP e os gargalos desse processo na visão dos feirantes. A metodologia consiste numa pesquisa descritiva, qualitativa que utilizou a técnica de observação, tendo como universo a feira-livre realizada no município de Porto Grande - AP. Os resultados indicaram que a capilaridade das Cadeias Curtas Agroalimentares, na região estudada envolve a articulação de atributos e valores que são associados ao produto comercializado e aos sujeitos participantes da transação. O estudo apontou ainda contribuições para melhorar a estruturação da feira livre no munícipio, visando transmitir melhores informações ao consumidor, bem como identificou os principais gargalos apontados pelos feirantes.
\end{abstract}

Palavras-chave: Agricultura familiar; Distribuição agroalimentar; Consumidores.

\begin{abstract}
The capillarity of short agrifood chains enables the generation of economic benefits for both producers and consumers. Therefore, this research aims to investigate the capillarity of the short agrifood chains, established at the family farming fair in the city of Porto Grande - AP and the bottlenecks of this process in the view of marketers. The methodology consists of a descriptive, qualitative research that used the observation technique, having as a universe the open-air market held in the city of Porto Grande - AP. The results indicated that the capillarity of the Short Agrifood Chains in the studied region involves the articulation of attributes and values that are associated with the marketed product and the subjects participating in the transaction. The study also pointed out contributions to improve the structure of the open market in the municipality, aiming to transmit better information to the consumer, as well as identifying the main bottlenecks pointed out by the marketers.
\end{abstract}

Keywords: Family farming; Agri-food distribution; Consumers.

\section{Resumen}

La capilaridad de las cadenas agroalimentarias cortas permite generar beneficios económicos tanto para los productores como para los consumidores. Por tanto, esta investigación tiene como objetivo investigar la capilaridad de las cadenas agroalimentarias cortas, instaladas en la feria de agricultura familiar de la ciudad de Porto Grande - AP y los cuellos de botella de este proceso a juicio de los comercializadores. La metodología consiste en una investigación descriptiva, cualitativa que utilizó la técnica de observación, teniendo como universo el mercado al aire libre realizado en la ciudad de Porto Grande - AP. Los resultados indicaron que la capilaridad de las Cadenas Agroalimentarias Cortas en la región estudiada implica la articulación de atributos y valores que se asocian con el producto comercializado y los sujetos que participan en la transacción. El estudio también señaló contribuciones para mejorar la estructura del mercado abierto en el municipio, con el objetivo de transmitir mejor información al consumidor, así como identificar los principales cuellos de botella señalados por los comercializadores.

Palabras clave: Agricultura familiar; Distribución agroalimentaria; Consumidores. 


\section{Introdução}

As mudanças sociais, políticas, técnicas, econômicas e culturais, levou ao surgimento de um industrializado sistema agroalimentar (Lopes et al., 2019), com predominância de uma estrutura globalizada das grandes empresas de processamento de alimentos e supermercados (Amaral et al., 2020). Esses fatores, indubitavelmente, alteraram a dinâmica relacional entre os consumidores, devido a pluralidade de atores que passaram a participar desse processo (Vale et al., 2020).

O padrão de industrialização alimentar compeliu fazendas ao processo de especialização da produção, com um padrão agroexportador, consequentemente os produtores delegaram o processamento de alimentos e distribuição para empresas especializadas fora das fronteiras da fazenda (Ochoa et al., 2019).

No entanto, este modelo está levantando preocupações e está sujeito a críticas em muitos pontos de vista, entre os quais difícil acesso ao mercado para pequenos proprietários e pequenas e médias empresas, poluição ambiental e ameaça à segurança alimentar e nutricional (Amaral et al., 2020).

A "revolução" instituída por iniciativas Cadeias Curtas Agroalimentares (CCAAs) ou Circuitos Curtos de Comercialização, especialmente na Europa e no Estados Unidos (Lopes et al., 2019), e mais recente nos estudos abordados no Brasil (Aguiar, DelGrossi, \& Thomé, 2018; Amaral et al., 2020; Renting, Marsden \& Banks, 2017; Vale et al., 2020), estão contribuindo para os objetivos da Agenda 2030 das Nações Unidas para a Sustentabilidade e redução da fome e pobreza.

Atuando no cenário das CCAAs, as feiras-livres coadjuvam neste contexto e caracterizam-se como formas alternativas de rede alimentar curta, incitando a produção dos alimentos nestas mesmas feiras, que refletem características dos produtores locais.

Assim, diversos estudos (Aguiar, DelGrossi, \& Thomé, 2018; Renting, Marsden \& Banks, 2017; Vale et al., 2020) foram iniciados no meio rural, por conta de uma nova política alimentar voltada para a satisfação de áreas negligenciadas pelas regulamentações governamentais convencionais, bem como pela crescente preocupação pública quanto à origem e manipulação dos alimentos.

Vale et al. (2020) realizaram um estudo de caso das feiras-livres presentes na cidade de Iporá-GO a partir dos conceitos aplicados às CCAAs. Aguiar et al. (2019) através da revisão bibliográfica, apontou as características das cadeias curtas com o fomento da agricultura familiar. Amaral et al. (2020) analisaram as medidas do circuito curto instituídas pela Central de Comercialização da Agricultura Familiar no Estado do Rio Grande do Norte, para prover o consumo sustentável na região semiárida.

Diante das evidências existentes sobre a relação entre às CCAAs e feiras de agricultura alimentar, no tocante as negligências governamentais, origem e manipulação dos alimentos e satisfação dos pequenos feirantes, esse estudo tem como objetivo: averiguar a capilaridade das cadeias curtas agroalimentares, instituída na feira de agricultura familiar no município de Porto Grande - AP e os gargalos desse processo na visão dos feirantes. A partir desse objetivo, leva-se à seguinte questão de pesquisa: Qual a capilaridade das cadeias curtas agroalimentares, instituída na feira de agricultura familiar no município de Porto Grande - AP e os gargalos desse processo na visão dos feirantes.

\section{Marco teórico}

\subsection{Conceito básico da agricultura familiar}

Sabe-se que a agricultura brasileira principalmente a agricultura familiar, contribui substancialmente para o mercado doméstico, produzindo alimentos a preços baixos, e colaborando para o crescimento local onde as famílias estão inseridas. O termo agricultura familiar é usado, no Brasil, em razão da referência realizada na Lei no $11.326 / 200$, em seu art. $3^{\circ}$.

[...] é considerado agricultor familiar e empreendedor familiar rural aquele que pratica atividades no meio rural, 
possui área de até quatro módulos fiscais, mão de obra da própria família, renda familiar vinculada ao próprio estabelecimento e gerenciamento do estabelecimento ou empreendimento pela própria família. Também são considerados agricultores familiares: silvicultores, aquicultores extrativistas, pescadores, indígenas, quilombolas e assentados da reforma agrária (MDA, 2019).

Sendo assim, para Matos e Marin (2009) os produtores familiares revelam grande capacidade adaptativa aos diferentes ambientes socioeconômicos, e essa reprodução social está intimamente relacionada às distintas e heterogêneas formas de estrutura social, cultural e econômica, introduzidas em um espaço é em dado contexto histórico. A agricultura familiar representa um dinamismo para a economia brasileira, principalmente no fornecimento de alimentos básicos para a população, produção esta que gera renda e emprego para a população rural, provendo a base econômica de diversos municípios brasileiros.

\subsection{Cadeias curtas agroalimentares (CCAAs) e a relevância das feiras livres no sistema agroalimentar}

Cadeias Curtas Agroalimentares (CCAAs) ou Circuitos Curtos de Comercialização foram originalmente identificadas como exemplos da resistência dos agricultores ao processo de modernização e, em seguida, à globalização do sistema alimentar (Van der Ploeg et al., 2000). Não é por acaso que a análise das cadeias de abastecimento curtas se desenvolveu sobretudo na literatura de 'Redes alternativas de alimentos' (Goodman, 2012) e na de sistemas agrícolas locais (Marsden et al., 2000).

A difusão das Cadeias Curtas Agroalimentares (CCAAs) ou Circuitos Curtos de Comercialização é um dos fenômenos mais evidentes no sistema agroalimentar, por representar diversas categorias de stakeholders, instituições públicas, cooperativas, associações e consumidor (Marsden \& Renting, 2017).

Em termos gerais, a CCAAs é caracterizada pela redução do elo direto entre a produção e o consumo (Lopes et al., 2019). Para Belletti e Marescotti (2013) a cadeia curta é constituída por um número limitado de operadores econômicos que se comprometem a promover a cooperação, o desenvolvimento local e o estreitamento sócio territorial das relações entre produtores e consumidores.

Para Marsden e Renting (2017), o elemento-chave que definem as cadeias curtas são embasadas nas dimensões organizacionais e participação horizontais, operando entre as duas pontas da cadeia alimentar (montante a jusante), permitindo que novas formas de governança alimentar sejam articuladas. Segundo Belleti e Marescotti (2013) a redução da distância geográfica que o produto percorre antes de antes de chegar ao consumidor, orientado pela busca de um produto de qualidade, mais fresco, aproximação entre produtores e consumidores e redução das ações intermediárias, é uma das características desse processo.

Por fim, um curto-circuito pode ser definido em termos de sua proximidade geográfica, relações sociais, vínculo atual entre o produtor e o consumidor, desenvolvimento local e incrustação de alimentos (Amaral et al., 2020). A capilaridade é tida com uma característica das cadeias curtas, na qual são instituídas estratégias para prover o seu desenvolvimento, expansão das vendas e produtos, fomentando assim o crescimento dos agricultores familiares bem como das feiras livres (Ochoa et al., 2019).

A relevância CCAAs baseia-se na consciência da insustentabilidade do sistema agroalimentar cada vez mais globalizado, com acesso aos alimentos com custos menores devido à economia de escala e especialização produtiva (Belleti \& Marescotti, 2013), entretanto, esse tipo de produção vem gerando indagações e novas reflexões sob a ótica econômica, ambiental, social, nutricional e qualidade alimentar (Lopes et al., 2019). Verano, Figueiredo e Medina (2021) apontam que as CCAAs são uma inovação social que permite os produtos dos pequenos produtores rurais e periurbanos resistirem à padronização dos alimentos e o encurtamento das relações de compra e venda. 
No que diz respeito aos impactos sociais, há exemplos claros de que a CCAAs pode gerar uma melhor distribuição do poder de negociação ao longo da cadeia de abastecimento: a distância social mais curta melhora a capacidade dos consumidores de adquirir informações, reduzindo a assimetria de informação (Lombardi et al., 2012), permitindo-lhes exercer um controle adequado sobre a governança da cadeia de abastecimento (Goodman, 2002).

Pesquisa apontam para os efeitos benéficos que as cadeias de suprimentos curtas podem produzir (King et al., 2010; Lopes et al., 2019).) como, por exemplo, mudanças no comportamento do consumidor. A cadeia de abastecimento curta pode contribuir para a melhoria da saúde de grupos vulneráveis, principalmente se utilizada estrategicamente para compra de produtos orgânicos e in natura. Elas atuam como um conjunto de ações para mitigar os efeitos gerados pela complexificação do sistema agroalimentar das grandes empresas, no que diz respeito as dimensões: sociais, espaciais, tecnológica e nutrição.

Cadeias Curtas Agroalimentares têm sido objeto de vários estudos, mas dificilmente analisadas do ponto de vista dos feirantes e da sua capilaridade. Do ponto de vista dos pequenos produtores, alguns estudos indicam que as principais barreiras enfrentadas pelas CCAAs em relação ao processo de distribuição envolvem a dispersão dos pontos de entrega e baixos volumes de vendas, os quais aumentam os custos do produto (Verano, Figueiredo \& Medina, 2021).

Outra questão destacada é que o setor nem sempre encontra demanda suficiente para a produção total e falta de canais de comercialização adequados para seus produtos. Nesse sentido, a motivação dos consumidores, assim como sua demanda por produtos, muitas vezes responde a critérios de diversidade, fato que pode colidir com a sazonalidade e limitações dos produtos em determinadas épocas do ano (Belleti \& Marescotti, 2013; Verano, Figueiredo \& Medina, 2021).

A cadeia de abastecimento curta representa uma inovação importante no que diz respeito ao sistema de distribuição agroalimentar dominante, especialmente em grandes áreas urbanas de consumo. No entanto, à luz do que foi discutido no parágrafo anterior, é necessária uma série de estudos que permitam averiguar os elos presentes nesse sistema, os gargalos existentes no processo de montante a jusante e ações que permitam o aumento da competitividade.

Nas últimas décadas, ocorreram mudanças importantes nas abordagens do desenvolvimento dos sistemas agroalimentares: o paradigma agroindustrial e o paradigma territorial. O paradigma agroindustrial está ancorado nos princípios do projeto de modernização agrícola que orientou o desenvolvimento dos sistemas agroalimentares. O paradigma territorial é voltado para sistemas agroalimentares sustentáveis (Kerton \& Sinclair, 2010).

As feiras livres fazem parte do segundo paradigma voltado ao desenvolvimento do território, pois elas podem ser definidas como iniciativas locais que visam integrar o a comercialização de produtos oriundos da agropecuária com a geração de renda e emprego em uma localidade (Morgan, 2008).

Para Gerhard e Peñaloza (2018) as feiras livres expressam o processo de ressocialização alimentar, reorganização da produção, distribuição e consumo, além de estar inserida dentro dos CCAAs (Morgan, 2008). Segundo King et al. (2010) o termo CCAAs configura o processo de produção-distribuição-consumo, como vendas diretas nas propriedades rurais, lojas especializadas, feiras livres e várias formas de grupos de compra.

Morgan (2008) acrescenta que as feiras livres são tidas como canais comerciais que visa a otimização do processo de produção e comercialização. Nesse processo o consumidor tem um papel mais ativo que nos sistemas convencionais de alimentação, pois ele tem as condições de averiguar as condições da origem do produto.

\section{Metodologia}

Quanto à abordagem do problema, realizou-se a análise qualitativa dos dados. Segundo (Astin, 2014) essa análise parte da perspectiva dos participantes, visando apenas a descrição das informações, uma vez que os dados coletados não foram tratados estatisticamente e, por fim, em relação às técnicas, foi aplicada a bibliográfica, através da análise das teorias que abarcam o tema exposto. 
A pesquisa identifica-se, quanto aos seus objetivos, como descritiva e exploratória, porque houve a investigação de fenômenos desconhecidos, cujas informações coletadas foram formalizadas (Marconi \& Lakatos, 2010). A referida pesquisa caracterizou-se dessa maneira em virtude à investigação de relato de experiência, da qual as informações coletadas foram de caráter qualitativo.

Utilizou-se a técnica de observação. A observação é um método de coleta de dados que envolve o pesquisador observando as interações no cenário natural (Greenhalgh \& Taylor, 1997), ocupando um papel relevante no ambiente analisado (Hsieh \& Shannon, 2005), produzindo uma descrição detalhada da interação no momento da pesquisa (Astin, 2014). Assim, o universo de pesquisa constitui-se da feira-livre realizada no município de Porto Grande - AP. Os dados coletados estão expostos no decorrer do trabalho.

\section{Resultados e discussão}

A estrutura das CCAAs no município de Porto Grande-AP representa um conjunto econômico, vinculado à relação entre os produtores, vendedores e consumidores. Ressalta-se que devido ao enfoque da pesquisa não identificou quantos feirantes são produtores, e sim, sua capilaridade e gargalos apontados pelos feirantes. A figura 1 representa a estrutura conceitural das Cadeias Curtas Agroalimentares (CCAAs) no município de Porto Grande-AP.

Figura 1: Estrutra conceitural das Cadeias Curtas Agroalimentares (CCAAs) no município de Porto Grande-AP.

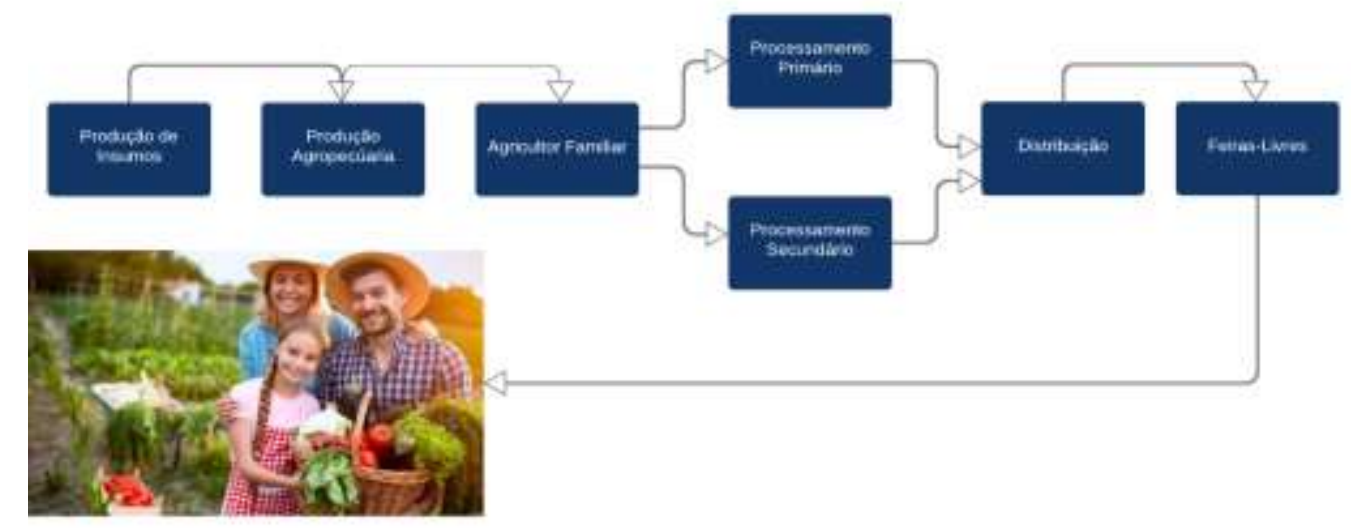

Fonte: Autores (2020).

Conforme a Figura 1, os aspectos de montante a jusante das CCAAs no munícipio de Porto Grande-AP, configura-se na compra de insumos que é realizada nas lojas agropecuárias do próprio município (antes da porteira), posteriormente o processo produtivo (dentro da porteira) é realizado nas propriedades familiares, onde, os próprios produtores plantam e comercializam nas feiras ou revendem para outros feirantes, alguns produtores são comercializados de forma in natura, ou recebem algum processamento secundário de forma artesanal, como por exemplo, queijo, doces e rapaduras. Esses produtos são transportados até a feira da agricultura familiar (Figura 2), onde são vendidos diretamente ao consumidor (depois da porteira). 
Figura 2: Feira da agricultura familiar em Porto Grande-AP.

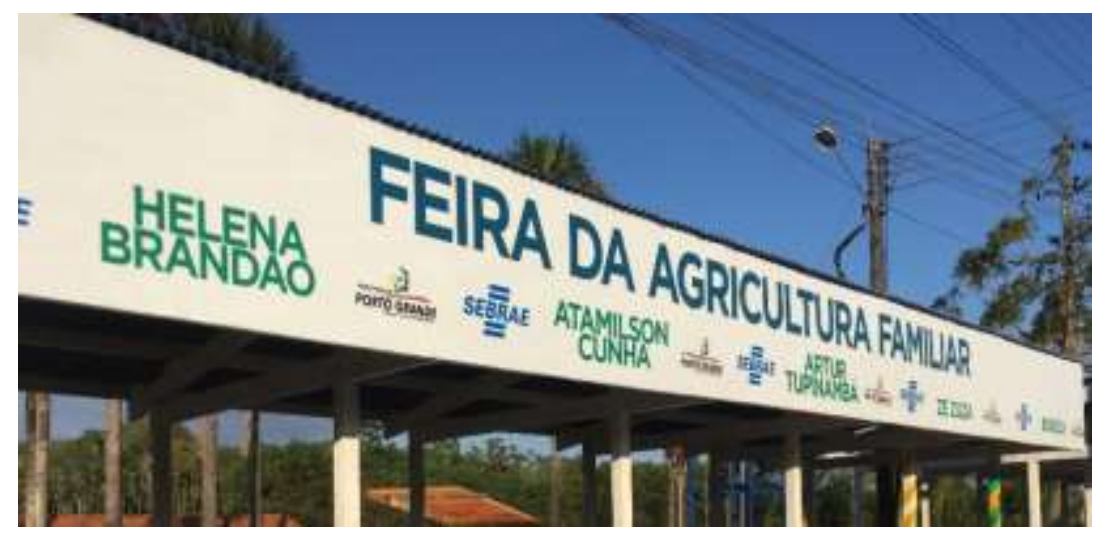

Fonte: Dados da pesquisa (2020).

Dessa forma, a capilaridade das CCAAs, na região estudada, consiste na articulação de atributos e valores, associados ao produto comercializado e aos sujeitos participantes da transação. Entretanto, a estruturação da feira livre no munícipio, pode melhorar essa articulação, com a transmissão de melhores informações ao consumidor, como o tipo de produção, monitorar o mercado, diferenciar mais seu produtor para reter o consumidor, marketing nos canais digitais, investimentos do poder público quanto aos aspectos de logística e capacitação técnica e assessoramento profissional de montante a jusante.

A feira da agricultura familiar em Porto Grande-AP, oferecem aos consumidores melhor acesso a produtos frescos e sazonais com menos impacto ao meio ambiente devido à redução na produção e no transporte associados aos alimentos locais. No entanto, a quantidade e a variedade de produtos oferecidos são limitadas, devido ao menor recursos para produção, processamento e transporte do que os grandes produtores, bem como menos poder de marketing e publicidade.

Portanto, a feira de agricultura familiar que é realizada no município de Porto Grande - AP, vem gerando empregos, beneficiando os pequenos produtores, como alternativa de renda, promovendo o desenvolvimento local e regional. De acordo com as informações prestadas pela presidente da feira, esses agricultores enfrentam algumas dificuldades que devem ser sanadas com uma assistência técnica e o melhor planejamento para o escoamento da produção, uma vez que a maior dificuldade desses produtores está no transporte desses produtos até a zona urbana do município de Porto Grande. A Associação conta com um caminhão apenas para atender os 50 feirantes semanalmente, o que inviabiliza o escoamento total do que é produzido.

Vale ressaltar também que a atividade no município enfrenta dificuldades quanto à assistência técnica, uma vez que o órgão responsável pela extensão rural no estado, não dispõe de recursos humanos e infraestrutura para atendimento das demandas existentes. Além disso, o acesso ao crédito nos bancos é restrito diante da burocracia existente, fato este que desestimula os produtores que manifestam a vontade de produzir, mas não tem recurso suficiente para investir no aumento da produção.

Os gargalos apontados pelos feirantes são:

- O questionamento de preço dos produtos por parte dos consumidores;

- Falta de produção diversificada;

- Ineficiência logística, o transporte não consegue atender as necessidades da associação, limitando a quantidade de produtos por produtor para comercialização, o que acaba prejudicando o escoamento total da produção;

- Falta de assistência técnica. 


\section{Conclusão}

A capilaridade das cadeias curtas agroalimentares, instituída na feira de agricultura familiar no município de Porto Grande - AP e os gargalos desse processo na visão dos feirantes configuram uma análise do desempenho da cadeia curta. Os gargalos enfrentados no processo de distribuição, produção e assistência técnica implicam uma modificação da posição e das funções desempenhadas pelos vários tipos de atores, para constituir alternativa às formas dominantes de distribuição nas feiras livres.

Como constituir outras formas de distribuição em áreas urbanas, como nos mercados, mercearias é até em hipermercados, ou seja, os vínculos criados horizontalmente os produtores/vendedores de forma horizontal e verticalmente com outros agentes da cadeia geram novas formas de organização nas cadeias curtas do munícipio.

Dessa forma, as CCAAs geram benefícios econômicos para produtores e consumidores, fortalecendo as relações sociais, preservando o ambiente, melhorando os aspectos nutricionais, fomentando o desenvolvimento local e preços mais justos para os agricultores, a menor ação de intermediários garantem que os agricultores obtenham uma fatia maior dos lucros.

Como sugestões para trabalhos futuros pode se realizar pesquisas envolvendo as CCAAs nas feiras livres de outros estados, visando comparar o desenvolvimento da agricultura familiar nos estados analisados.

\section{Referências}

Aguiar, L. C., DelGrossi, M. E., \& Thomé, K. M. (2018). Short food supply chain: characteristics of a family farm. Ciência Rural, $48(5)$, e20170775. https://www.scielo.br/j/cr/a/GmmnrGBJN7WJB68gKBYjn5b/?lang=en

Amaral, L. S., Santos, C. J., Rozendo, C., Penha, T. A. M., \& Araújo, J. P. (2020). O papel das Cadeias Curtas de Comercialização na construção de um modelo de desenvolvimento rural sustentável no semiárido nordestino: o caso da Central de Comercialização da Agricultura Familiar do Rio Grande do Norte (CECAFES). Desenvolv. Meio Ambiente, 55, 494-516. https://revistas.ufpr.br/made/article/view/74160/42394

Astin, F. (2014). Characteristics of qualitative research and its application. British Journal of Cardiac Nursing. February. 9(2). https://www.mag onlinelibrary.com/doi/abs/10.12968/bjca.2014.9.2.93

Belletti, G., \& Marescotti, A. (2003). Potenzialitá e limiti dele iniziative di filiera corta. Progress in nutrition, 15(3), 146-162. https://flore.unifi.it/handle/2158/822663\#.YTy53p1KjIU

Gerhard, F., \& Peñaloza, V. (2018). Resilience in trade fairs: a study in brazilian context. Interações (Campo Grande), 19(4), 855-869. https://www.scielo.br/j/inter/a/DRN3bFMDz4WFgx5pMxnQWgJ/?format=pdf\&lang=en

Greenhalgh, T., \& Taylor, R. (1997). How to read a paper: papers that go beyond numbers (qualitative research). BMJ, 315(7110), 740-3. https://www.bmj.com/content/315/7110/740

Goodman D. (2002). Rethinking food production-consumption: integrative perspectives. Sociologia Ruralis, $42 \quad$ (4), $271-277$. https://onlinelibrary.wiley.com/doi/10.1111/1467-9523.00216

Hsieh, H. F., \& Shannon, S. E. (2005). Three approaches to qualitative content analysis. Qual Health Res, 15(9), 1277-88. https://pubmed.ncbi.nlm.nih.gov/16204405/

Kerton, S., \& Sinclair, A. J. (2010). Buying local organic food: A pathway to transformative learning. Agriculture and Human Values, 27, 401-413. https://link.springer.com/article/10.1007/s10460-009-9233-6

King R. P., Hand M. S., DiGiacomo G., Clancy K., Gomez M. I., Hardesty S. D., Lev L., \& McLaughlin E. W. (2010). Comparing the Structure, Size, and Performance of Local and Mainstream Food Supply Chains. Usda Economic Research Service, 81. https://www.ers.usda.gov/publications/pubdetails/?pubid $=46407$

Ministério de Desenvolvimento Agrário - MDA. O que é agricultura familiar. http://www.mda.gov.br/sitemda/noticias/o-que-\%C3\%A9-agricultura-Familiar

Lombardi A., Pascucci S., Cembalo L., \& Dentoni D. (2012). Elementi organizzativi e governance delle reti alimentari comunitarie (Food Community Networks). Agriregionieuropa, 8 (29), 59. https://agriregionieuropa.univpm.it/it/content/article/31/29/elementi-organizzativi-e-governance-delle-retialimentari-comunitarie-food

Lopes, I. D., Basso, D., \& Brum, A. L. (2019). Cadeias agroalimentares curtas e o mercado de alimentação escolar na rede municipal de Ijuí, RS. Interações (Campo Grande), 20(2), 543-557. https://www.scielo.br/j/inter/a/6Vr36CBZYtczfRxH4CrYJzs/?lang=pt

Marsden T., Banks J., \& Bristow G. (2000). Food supply chain approaches: Exploring their role in rural development. Sociologia Ruralis, 40(4), 424-438. https://onlinelibrary.wiley.com/doi/abs/10.1111/1467-9523.00158 
Research, Society and Development, v. 10, n. 12, e143101220163, 2021

(CC BY 4.0) | ISSN 2525-3409 | DOI: http://dx.doi.org/10.33448/rsd-v10i12.20163

Marsden, T. \& Renting, H. (2017). Compreendendo as redes alimentares alternativas: o papel de cadeias curtas de abastecimento de alimentos no desenvolvimento rural. In: Gazolla, M. \& Schneider, S. (Eds), Cadeias curtas e redes agroalimentares alternativas: negócios e mercados da agricultura familiar. 59-82. Porto Alegre: UFRGS.

Matos, G. R. \& Marin, J. O. B. (2009). Agricultores familiares e sistemas de produção de frutas em Itapuranga, Goiás. Revista Pesquisa. 39(3), 197-206. https://www.revistas.ufg.br/pat/article/view/4096/5268

Morgan, K. (2008). Greening the realm: Sustainable food chains and the public plate. Regional Studies, 42, 1237-1250. https://www.tandfonline.com/doi/abs/10.1080/00343400802195154

Ochoa, C. Y., Matarán, A., Olmo, R. M., López, J. M. \& Fuentes-Guerra, R. (2019). The Potential Role of Short Food Supply Chains in Strengthening Periurban Agriculture in Spain: The Cases of Madrid and Barcelona. Sustainability, (11). https://www.mdpi.com/2071-1050/11/7/2080

Vale, N. K. A., Santana, S. N., Souza, C. B., \& Bottega, D. B. (2020). Short distribution channels of horticulture produce in Open-air markets in the City of Iporá-GO, Brasil. Research, Society and Development, $9(7), 1-15$, e223974035. https://rsdjournal.org/index.php/rsd/article/view/4035

Van der Ploeg J. D., Renting H., Brunori G., Knickel K., Mannion J., Marsden T., de Roest K., Sevilla-Guzman E., \& Ventura F. (2000). Rural development: From practices and policies towards theory. Sociologia Ruralis, 40(4), 391-408. https://onlinelibrary.wiley.com/doi/abs/10.1111/1467-9523.00156

Verano, T. de C., Figueiredo, R. S., \& Medina, G. da S. (2021). Agricultores familiares em canais curtos de comercialização: uma análise quantitativa das feiras municipais. Revista de Economia e Sociologia Rural, 59(3), e228830. https://www.scielo.br/j/resr/a/h MLL8mZFhGLTcHhnhJfjpQr/? ang=pt\#: :text=A\%20partir\%20de\%20dados\%20quantitativos,como\%20alternativa\%20ao\%20sistema\%20agroalimentar 